\section{Military Technical College Kobry El-kobbah, Cairo, Egypt}

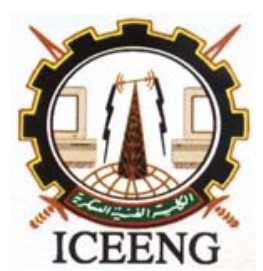

\title{
Real Time Implementation Of A Matched Filter For Radar Applications Based On Wave Digital Filters
}

\author{
K.H. Moustafa ${ }^{*}$ and Alaa Fahmy ${ }^{* * *}$
}

\begin{abstract}
Based on the theory of wave digital filters (WDFs), new model is derived to represent matched filter for radar applications, derived from lumped/distributed ladder networks. This model contains lumped elements and commensurate transmission lines. This paper also introduced a real-time implementation of the proposed model. We will demonstrate that this approach can be implemented in real time, although with this prototype the frequencies are too low for practical radar applications. The real-time implementation is performed based on input/output data acquisition card using MATLAB, SIMULINK toolbox, Real-Time Workshop (RTW) toolbox and Real-Time Windows target (RTWT) toolbox. In addition, real-time windows target requires the Watcom $\mathrm{C} / \mathrm{C}++$ compiler.
\end{abstract}

\section{INTRODUCTION}

Networks containing lumped and distributed elements have been dealt with by numerous authors [1-4]. In [4], the design and performance of ladder networks containing both lumped and distributed elements were described. Also a suitable lowpass to band-pass transformation was been developed.

The matched filter requires $\sin (x) / x$ frequency response, where $x=\left(\omega-\omega_{0}\right) \delta / 2, \omega_{0}$ is the central frequency and $\delta$ is the pulse width. We use distributed elements to realize the zeroes of transmission of the frequency response.

There is an increasing need for high-speed real time digital filters for applications such as video processing, and radar signal processing. WDFs have well known properties, which make them attractive for this task [5-7]. These filters exhibit good dynamic range performance and low sensitivity that make them suitable for use with very short coefficient wordlengths. In this paper, the design of WDF for a model of band-pass lumped/distributed matched filter is described. The proposed realization of WDF is simulated using the MATLAB simulator.

Real time implementation requires that the model is free of algebric loops (delay free loops). In computer time implementations, models with algebric loops are solved iteratily using an optimisation subroutine. This is not possible in real time. For this

\footnotetext{
${ }_{* * *}^{*}$ Egyptian Armed Forces

Egyptian Armed Forces
} 
reason, we introduced the WDF model in this paper as WD model avoid delay free loops. We present a real-time implementation of the SIMULINK model of a Lumped/distributed BPWDMF with commensurate transmission lines. This model is excited by a pulsed RF signal with frequency $f_{0}=500 \mathrm{rad} \mathrm{s}^{-1}$ and a pulse width $\delta=$ $0.28 \mathrm{~s}$. These parameters were chosen for the real-time realization purposes. These SIMULINK model is running at $10 \mathrm{KHz}(0.0001 \mathrm{~s})$. We should take into account that the maximum sample rates for a minimum model build using SIMULINK should not exceed $20 \mathrm{KHz}(0.00005 \mathrm{~s})$ in spite of the fact that the capability of the used card (Lab-PC-1200 National instruments data acquisition card) is up to $100 \mathrm{KHz}$ sample rate.

\section{REALIZATION USING LUMPED AND DISTRIBUTED LADDER NETWORK}

In this paper, we design a radar with the following parameters; central frequency $\omega_{0}=500 \mathrm{rad} \mathrm{s}^{-1}$, and pulse width $\delta=0.28 \mathrm{~s}$. From the above data, the bandwidth is given by $\Delta \omega=2 \pi \Delta f=30.74272811 \mathrm{rad} \mathrm{s}^{-1}$ [7]. Then, we obtain the following parameters: $\omega_{1}=484.864859 \mathrm{rad} \mathrm{s}{ }^{-1}, \omega_{2}=515.6075817 \mathrm{rad} \mathrm{s}^{-1}$, where $\omega_{1}$ and $\omega_{2}$ are two cut-off frequencies of the band-pass filter respectively.

Based on [6], we start with the third-order prototype lumped low-pass filter, shown in Figure.1, but with different values for shunt capacitors $C_{1}$ and $C_{2}$. Open-ended transmission lines with different delay times $T_{1}$ and $T_{2}$ replace these capacitors. From the zeros of the $\sin (x) / x$ response, we determine the values of the delays $T_{1}$ and $T_{2}$. This is followed by an optimization step to calculate the values of the characteristic impedances $Z_{1}$ and $Z_{2}$ of the transmission lines and the value of the inductance $L$.

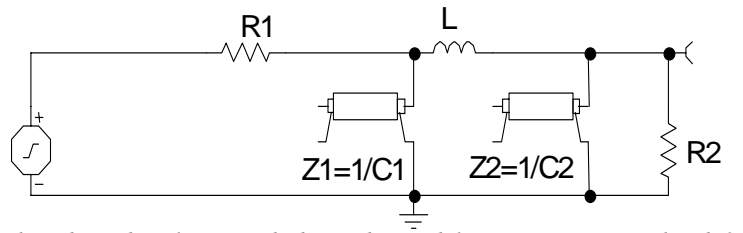

Figure 1. Third order lumped/distribute dow-pass matched filter.

The low-pass to band-pass filter transformation is made based the steps derived in [6]. This results a third order lumped/distributed band-pass matched filter with commensurate transmission lines. The resulting filter is shown in Figure 2.

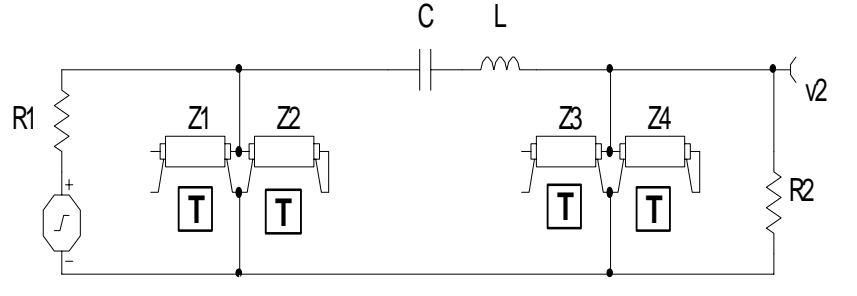

Figure 2. Third order lumped/distributed band-pass matched filter with commensurate transmission lines.

This band-pass filter has the following parameters: $R_{1}=R_{2}=50 \Omega, L=3.507 \mathrm{H}$, $C=1.14 \mu \mathrm{F}, \quad Z_{1}=97.0597 \Omega, \quad Z_{2}=107.41924 \Omega, \quad Z_{3}=143.035425 \Omega$, and $Z_{4}=$ 
$158.3021 \Omega$. In this case all transmission lines have the same delay time $T=0.020369$ sec.

\section{REALIZATION OF FILTER CIRCUITS USING WAVE DIGITAL APPROACH}

As shown in Figure 2, this filter represents a doubly terminated ladder network consisting of lumped elements and commensurate transmission lines. Its construction using WDF is shown in Figure 3.

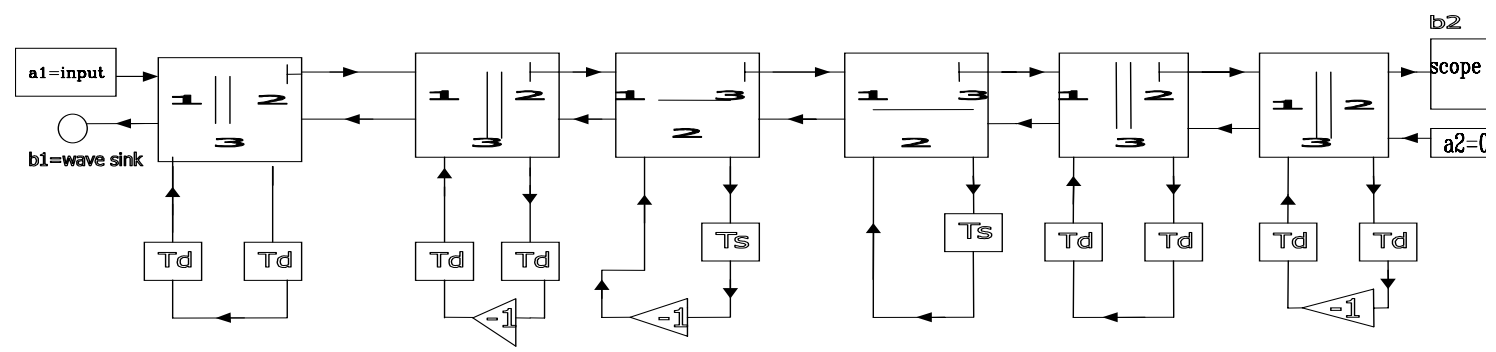

a) Structure of the third order lumped/distributed with commensurate transmission lines band-pass matched WDF.

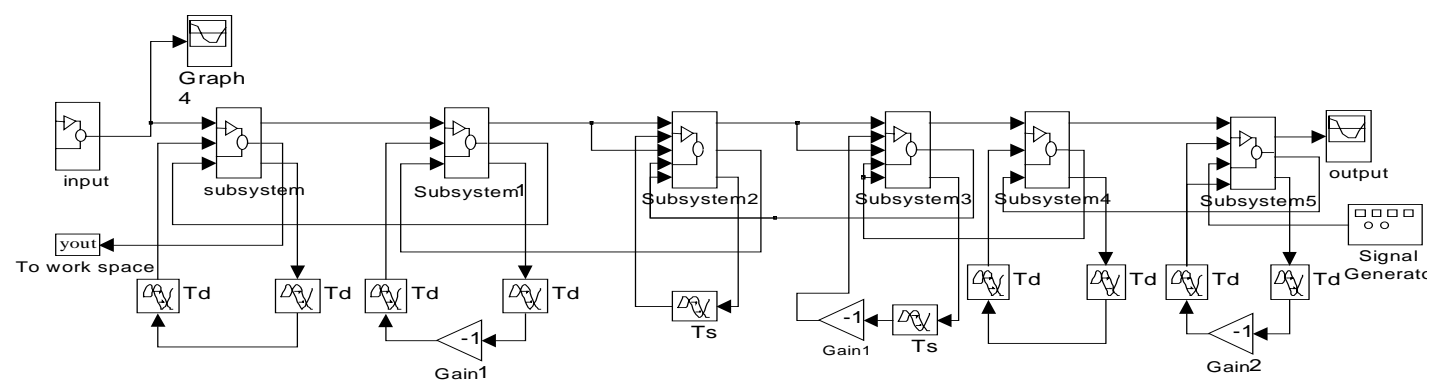

(b) Realization using Simulink and MATLAB

Figure 3. WDF representation of the filter of Figure 2.

The description of the subsystems shown above in Figure 3(b) and the values of multiplication coefficients of adaptors are shown at Table (1). The sample time of the filter was chosen to satisfy the sampling theorem [8] such that $f_{\mathrm{s}} \geq f_{0}$.

\begin{tabular}{|c|c|c|}
\hline No. & Description & $\begin{array}{l}\text { Multiplication } \\
\text { coefficient }\end{array}$ \\
\hline Subsystem & $\begin{array}{l}\text { Three-port parallel adaptor, port- } 2 \text { is the } \\
\text { reflection free, and port } 3 \text { is the dependent port }\end{array}$ & $\gamma_{0}=-0.339997837$ \\
\hline $\begin{array}{l}\text { Subsystem } \\
1\end{array}$ & $\begin{array}{l}\text { Three-port parallel adaptor, port- } 2 \text { is the } \\
\text { reflection free, and port } 3 \text { is the dependent port }\end{array}$ & $\gamma_{1}=-0.235010939$ \\
\hline $\begin{array}{l}\text { Subsystem } \\
2\end{array}$ & $\begin{array}{l}\text { Three-port series adaptor, port- } 3 \text { is the } \\
\text { reflection free, and port } 2 \text { is the dependent port }\end{array}$ & $\gamma_{2}=0.05722857$ \\
\hline $\begin{array}{l}\text { Subsystem } \\
3\end{array}$ & $\begin{array}{l}\text { Three-port series adaptor, port- } 3 \text { is the } \\
\text { reflection free, and port } 2 \text { is the dependent port }\end{array}$ & $\gamma_{3}=0.062028083$ \\
\hline $\begin{array}{l}\text { Subsystem } \\
4\end{array}$ & $\begin{array}{l}\text { Three-port parallel adaptor, port- } 2 \text { is the } \\
\text { reflection free, and port } 3 \text { is the dependent port }\end{array}$ & $\gamma_{4}=-0.981231441$ \\
\hline $\begin{array}{l}\text { Subsystem } \\
5\end{array}$ & $\begin{array}{l}\text { Three-port parallel adaptor, port-3 is the } \\
\text { dependent port }\end{array}$ & $\begin{array}{l}\gamma_{51}=0.42611052 \\
\gamma_{52}=1.196099567\end{array}$ \\
\hline
\end{tabular}


Table 1. Multiplication coefficients and description of adaptors of WDMF in Figure 3

We now check the matching of this filter by applying an input as a pulsed RF signal with a frequency $\omega_{0}=500 \mathrm{rad} \mathrm{s}^{-1}$, and pulse width $\delta=0.28 \mathrm{sec}$. It is clear from Figure 4 that the maximum output generated by this filter occurs at the delay time $\delta$ which indicates the completion of the arrival of input signal.
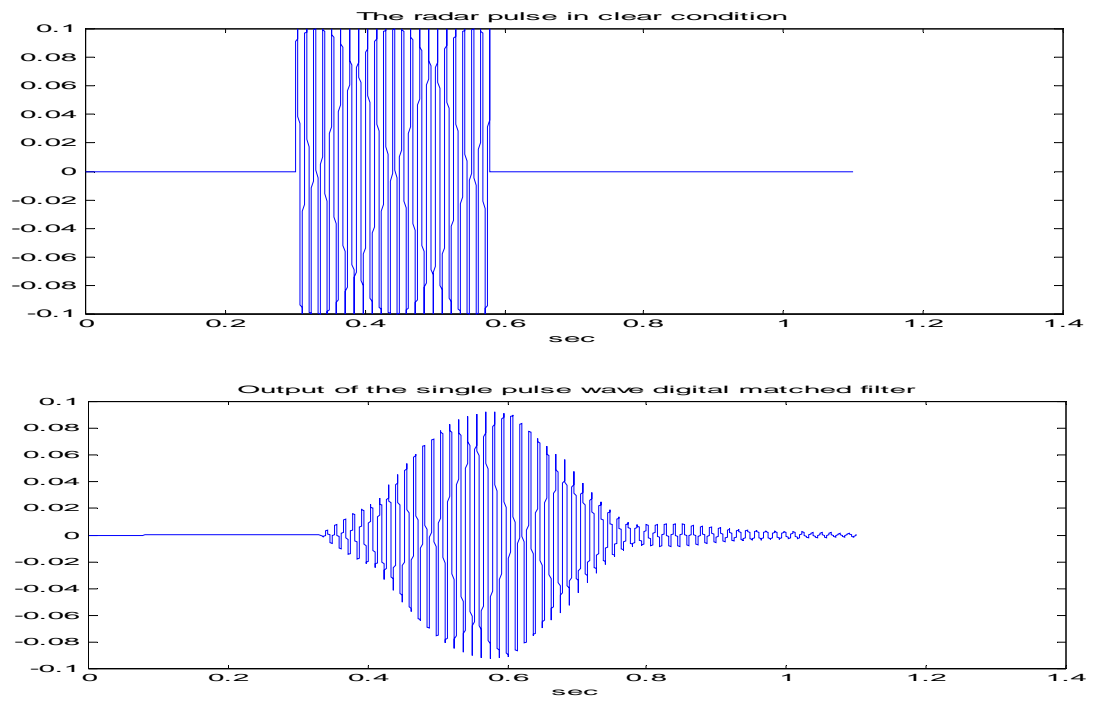

Figure 4. Output of band-pass with commensurate transmission lines filter matched to a rectangular pulse.

\section{REAL-TIME IMPLEMENTATION OF THIRD ORDER LUMPED/DISTRIBUTED WITH COMMENSURATE TRANSMISSION LINES BAND-PASS MATCHED WDF}

The real-time implementation is based on input/output data acquisition card using MATLAB, SIMULINK, the RTW toolbox and the RTWT toolbox. In addition, RTWT requires the Watcom $\mathrm{C} / \mathrm{C}++$ compiler. Real time implementation requires that the model is free of algebric loops (delay free loops). In computer time implementations, models with algebric loops are solved iteratily using an optimisation subroutine. This is not possible in real time. The introduced WDMF model is excited by a pulsed RF signal with frequency $f_{0}=500 \mathrm{rad} \mathrm{s}^{-1}$ and a pulse width $\delta=0.28 \mathrm{~s}$. The SIMULINK model is running at $10 \mathrm{KHz}(0.0001 \mathrm{~s})$. We should take into account that the maximum sample rates for a minimum model build using SIMULINK should not exceed $20 \mathrm{KHz}(0.00005 \mathrm{~s})$ in spite of the fact that the capability of the used card (Lab-PC-1200 National instruments data acquisition card) is up to $100 \mathrm{KHz}$ sample rate.

The process of creating and running a real-time application are; creating a SIMULINK model, entering simulation parameters for real-time workshop, entering scope properties for signal tracing, creating a real-time application, connecting the SIMULINK model to the real-time application, and running a real-time application.

The RTW provides the utilities for converting SIMULINK models into C code, and then compiling the code into a real time executable. The executable is then loaded into memory and the RTWT runs the executable in real time. The RTWT uses a small 
real-time kernel to ensure that the real-time application runs in real time. The realtime kernel runs at PC clock as its primary source of time. It is only during real-time execution of the model that the kernel intervenes to ensure that your model is given priority to use CPU to execute each model update at the prescribed sample intervals. Once the model update at a particular sample interval completes the kernel releases the CPU to run any other windows application that may need servicing. The kernel interfaces and communicates with I/O hardware using I/O drivers. It checks for proper installation of the $\mathrm{I} / \mathrm{O}$ board using plug and play. If the board has been properly installed, the drivers allow the real-time application to run.

Integration between SIMULINK external mode and the RTWT enables capturing and displaying signals from the real-time application. During real-time execution, SIMULINK's external mode provides bi-directional exchange of data. New model parameter value values can be passed down to the real-time application while signal data can be retrieved from the real-time application and displayed in SIMULINK scope blocks. SIMULINK external mode provides a universal interface for use with RTW targets. It allows us to make parameter changes in the SIMULINK block diagram and have the new parameter changes exported automatically to the real-time application. External mode requires a communication interface to pass parameters external to SIMULINK, and on the receiving end, the same communications protocol must be used to accept new parameter values and insert them in the proper memory locations for use by the real-time application. In the case of RTWT, the host computer also serves as the target computer. Therefore, only a virtual device driver is needed to exchange parameters between MATLAB and SIMULINK memory space and memory that is accessible by the real-time application.

The RTWT provides drivers for different boards. These drivers connect the physical world to the real-time application. Connecting sensors and actuators to I/O boards does this connection to the physical world. The drivers then read data from and write data to I/O boards.

The adapter block is a special block that is not connected to any other blocks in the SIMULINK block diagram. The purpose of the adapter block is to provide a reference to a particular I/O board. After we add an adapter block to our SIMULINK model, we can configure the I/O driver to match settings on the physical I/O board, and selecting settings that are software programmable. The RT In and RT Out blocks provide an interface to our physical I/O board. They ensure that the $\mathrm{C}$ code generated with realtime workshop correctly maps block diagram signals to the appropriate I/O channels. The RTWT uses the RT In block for all input signals: analog inputs, and digital inputs. The RT Out block handles all output signals: analog outputs, and digital outputs.

The real-time model of lumped/distributed BPWDMF with commensurate transmission lines is shown in Figure 5

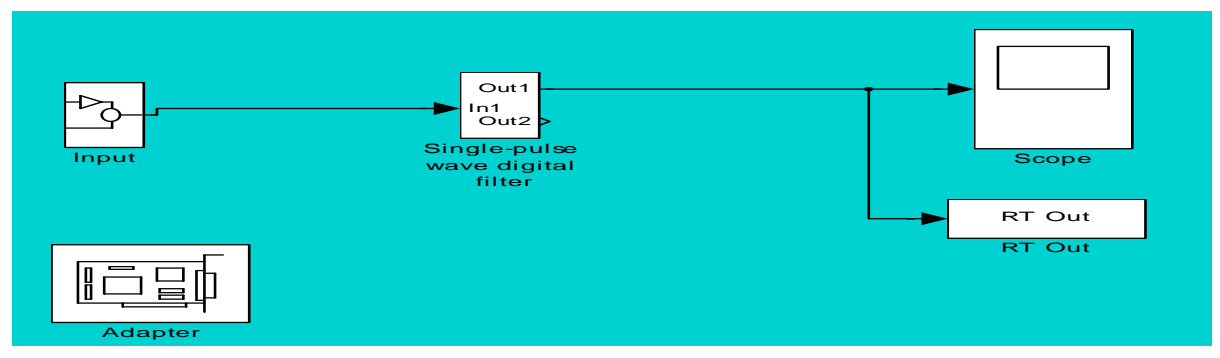


Figure 5. Real-time model of a lumped/distributed BPWDMF with commensurate transmission lines

The real-time representation of both the input and output of the WDF mentioned above is shown in Figure 6.

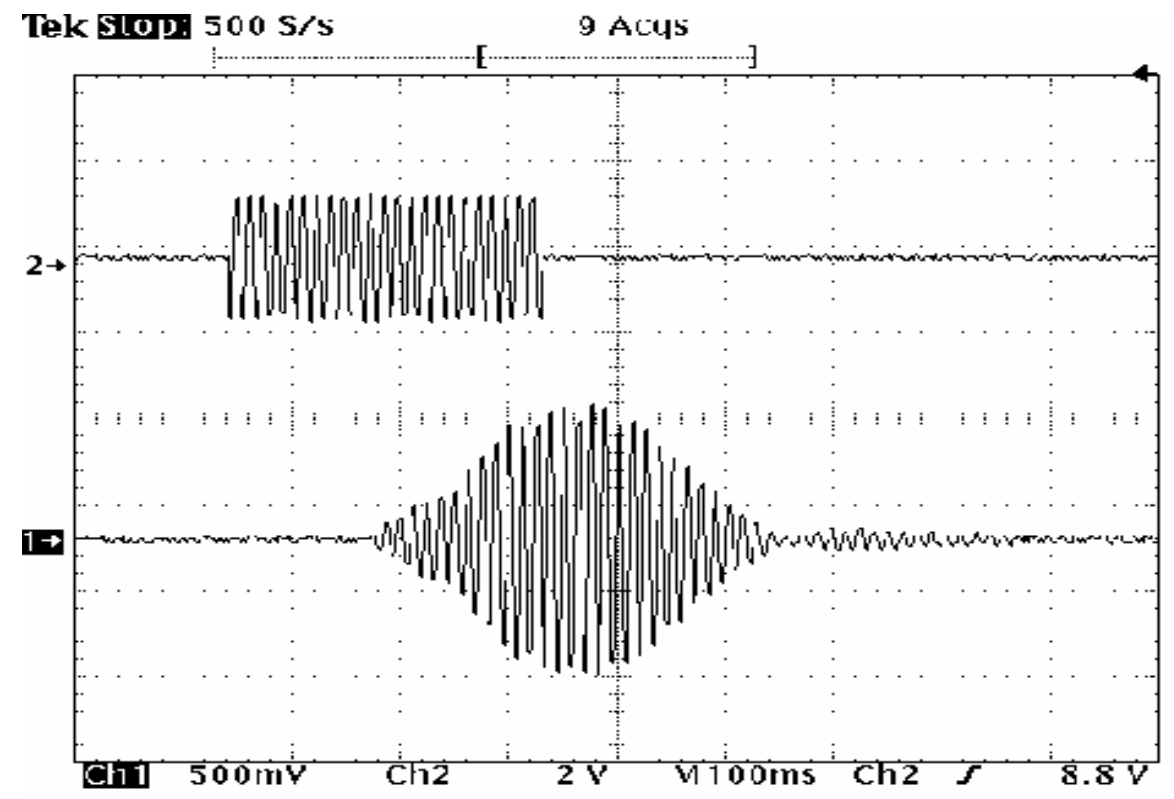

Figure 6 Real-time representation of both the input and output of a lumped/distributed $B P W D M F$ with commensurate transmission lines

\section{CONCLUSION}

We have presented a design procedure for the construction of matched filter for radar applications derived from lumped/distributed ladder networks.

The procedure has the following advantages:

- The design circuits can accurately approximate the $\sin (x) / x$ frequency response.

- $\quad$ The procedure is simple to apply.

We have also presented the wave digital filter model for circuit contains commensurate transmission lines. This model is built using SIMULINK.

This paper also introduces a real-time implementation of the proposed wave digital filter model. The real-time implementation is achieved based on Lab-PC-12000 National instrument input/output data acquisition card using MATLAB, SIMULINK, the real-time workshop toolbox, and the real-time windows target toolbox. The measurement result has a good agreement with that obtained from the simulation model. 


\section{REFERENCES}

1. T. Koga, "Synthesis of finite passive n-ports with prescribed two variable reactance matrices”, IEEE Trans. Circuit Theory, Vol.CT-13, pp.31-52, Mar.1966.

2. D. C. Youla, "The synthesis of networks containing lumped and distributed elements”, in Proc. Symp. generalized Networks, Brooklyn, pp. 289-343, Apr.1966.

3. J. D. Rhodes and P. C. Marston, "Cascade synthesis of two-variable one-elementkind networks”, IEEE Trans. Circuit Theory, Vol.CT-19, pp.78-80, Jan.1972.

4. M.I.Sobhy, and Mootaman Safi, "The design of a class of microwave filters using lumped and Distributed Elements", IEEE Trans. on microwave theory and techniques, Vol.MTT-25, No.12, December 1977.

5. Lfred Fettweis and Klaus Meerkötter, “On adaptors for wave digital filters”, IEEE transactions on acoustic, speech, and signal processing, Vol.ASSP-23, No.6, December 1975.

6. Fettweis, A.," Wave digital filters: theory and practice”, Proceedings IEEE, Vol. 74, 270- 327, February 1986.

7. Gazsi, L., “Explicit formulas for lattice wave digital filters”, IEEE trans. Circuit \& Sys., Vol.32, 68-88, 1985. 